\title{
Some Results on Equi Independent Equitable Dominating Sets in Graph
}

\author{
S. K. Vaidya ${ }^{1 *}$, N. J. Kothari ${ }^{2}$ \\ ${ }^{1}$ Department of Mathematics, Saurashtra University, Rajkot 360005, Gujarat, India
}

Received 18 May 2015, accepted in final revised form 26 June 2015

\begin{abstract}
A subset $D$ of $V(G)$ is called an equitable dominating set if for every $v \in V(G)-D$, there exists a vertex $u \in D$ such that $u v \in E(G)$ and $|\operatorname{deg}(u)-\operatorname{deg}(v)| \leq 1$. A subset $D$ of $V(G)$ is called an equitable independent set if for any $u \in D, v \notin N^{e}(u)$ for all $v \in D-\{u\}$ where, $N^{e}(u)=\{v \in V(G) / v \in N(u),|\operatorname{deg}(u)-\operatorname{deg}(v)| \leq 1\}$. An equitable dominating set $D$ is said to be an equi independent equitable dominating set if it is also an equitable independent set. The minimum cardinality of an equi independent equitable dominating set is called equi independent equitable domination number which is denoted by $i^{e}$. We investigated an equi independent equitable domination number for some special graphs.
\end{abstract}

Keywords: Equi independent equitable domination number; Equitable domination number; Domination number.

(C) 2015 JSR Publications. ISSN: 2070-0237 (Print); 2070-0245 (Online). All rights reserved.

doi: http://dx.doi.org/10.3329/jsr.v7i3.23412 J. Sci. Res. 7 (3), 77-85 (2015)

\section{Introduction}

Throughout this work, the term graph we mean finite, connected, undirected and simple graph $G$ with vertex set $V(G)$ and edge set $E(G)$. For any undefined term we rely upon West [9] and Haynes et al. [5]. For every vertex $v \in V(G)$ the open neighbourhood set $N(v)$ is the set of all vertices adjacent to $v$ in $G$. That is, $N(v)=\{u \in V(G) / u v \in E(G)\}$. The closed neighbourhood set $N[v]$ of $v$ is defined as $N[v]=N(v) \bigcup\{v\}$. A set $D \subseteq V(G)$ is called a dominating set if every vertex in $V(G)-D$ is adjacent to at least one vertex in $D$. The domination number $\gamma(G)$ is the minimum cardinality of a dominating set of $G$. A subset $D$ of $V(G)$ is an independent set if no two vertices in $D$ are adjacent. A dominating set $D$ which is also an independent set is called an independent dominating set. The independent domination number $i(G)$ is the minimum cardinality of an independent dominating set. The concept of an independent domination was formalized by Berge [2] and Ore [6] while the definition of an independent domination number and the notation

\footnotetext{
Corresponding author: samirkvaidya@yahoo.co.in
} 
$i(G)$ were introduced by Cockayne and Hedetniemi [3]. A survey on the concept of an independent domination can be found in Goddard and Henning [4] while applications of dominating sets in computer network is well studied by Basavanagoud and Hosamani [1]. A subset $D$ of $V(G)$ is called an equitable dominating set if for every $v \in V(G)-D$ there exists a vertex $u \in D$ such that $u v \in E(G)$ and $|\operatorname{deg}(u)-\operatorname{deg}(v)| \leq 1$. The minimum cardinality of such an equitable dominating set is equitable domination number of $G$ which is denoted by $\gamma^{e}$. A vertex $u \in V(G)$ is degree equitable adjacent or equitable adjacent with a vertex $v \in V(G)$ if $|\operatorname{deg}(u)-\operatorname{deg}(v)| \leq 1$ for $u v \in E(G)$. An equitable dominating set $D$ is said to be a minimal equitable dominating set if no proper subset of $D$ is an equitable dominating set. Swaminathan and Dharmalingam [7] have derived following necessary and sufficient condition for minimal equitable dominating set.

Theorem 1.1: An equitable dominating set $D$ is minimal if and only if for every vertex $u \in D$ one of the following holds.

(i) Either $N(u) \cap D=\varnothing$ or $|\operatorname{deg}(v)-\operatorname{deg}(u)| \geq 2$ for all $N(u) \cap D$.

(ii) There exists a vertex $v \in V(G)-D$ such that $N(v) \cap D=\{u\}$ and $|\operatorname{deg}(v)-\operatorname{deg}(u)| \leq 1$.

A vertex $v \in V(G)$ is called equitable isolate if $|\operatorname{deg}(v)-\operatorname{deg}(u)| \geq 2$ for every $u \in N(v)$. It is obvious that, if $v \in V(G)$ is an equitable isolate and $D$ is any equitable dominating set then $v \in D$. Obviously isolated vertices are equitable isolates. Hence $I_{s} \subseteq I_{e} \subseteq D$ for every equitable dominating set $D$, where $I_{s}$ and $I_{e}$ are the set of all isolated vertices and set of all equitable isolates of $G$ respectively. As reported in [7], a graph $G$ has a unique minimal equitable dominating set if and only if the set of all equitable isolates form an equitable dominating set.

The equitable neighbourhood of $v$ denoted by $N^{e}(v)$ is defined as $N^{e}(v)=\{u \in V(G) / u \in N(v),|\operatorname{deg}(u)-\operatorname{deg}(v)| \leq 1\}$. The cardinality of $N^{e}(v)$ is denoted by $\operatorname{deg}_{G}^{e}(v) . \Delta^{e}(G)=\max _{v \in V(G)}\left|N^{e}(v)\right|$, and $\delta^{e}(G)=\min _{v \in V(G)}\left|N^{e}(v)\right|$ are known as maximum and minimum equitable degree of graph $G$ respectively.

Remark 1.2: $\delta^{e}(G) \leq \delta(G)$ and $\Delta^{e}(G) \leq \delta(G)$.

Remark 1.3: In regular graphs and $(k, k+1)$ bi-regular graphs, $\delta^{e}(G)=\delta(G)$ and $\Delta^{e}(G)=\delta(G)$.

Remark 1.4: $\Delta^{e}(G)=\delta^{e}(G)=0$ for $K_{1, n}$ for $n \geq 3$.

Swaminathan and Dharamlingam [7] have also introduced the concept of equitable independent set. According to them a subset $D$ of $V(G)$ is called an equitable independent set if for any $u \in D, \quad v \notin N^{e}(u)$ for all $v \in D-\{u\}$. The maximum cardinality of an equitable independent set is denoted by $\beta^{e}$.

Remark 1.5: Every independent set is an equitable independent set. 
Remark 1.6: [7] Let $D$ be a maximal equitable independent set. Then $D$ is a minimal equitable dominating set.

Motivated by the concept of equitable dominating set and equitable independent set a new concept was conceived by Swaminathan and Dharamlingam [7] while it was formalized and named as equi independent equitable dominating set by Vaidya and Kothari [8].

Definition 1.7: An equitable dominating set $D$ is said to be equi independent equitable dominating set if it is also equitable independent set. The minimum cardinality of an equi independent equitable dominating is called equi independent equitable domination number which denoted by $i^{e}$.

Illustration 1.8: In Fig. 1 and $D=\left\{v, v_{1}, v_{5}, v_{8}, u_{3}, u_{7}, u_{9}\right\}$ is equitable independent set as well as equitable dominating set for closed helm $\mathrm{CH}_{9}$ with $i^{e}\left(\mathrm{CH}_{9}\right)=7$.

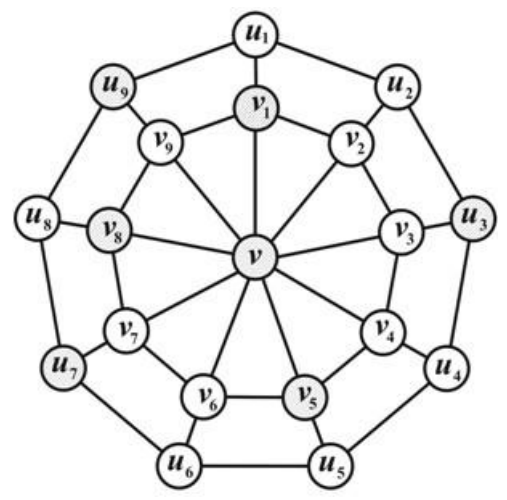

Fig. 1

\section{Main Results}

Definition 2.1: The corona $G_{1} \odot G_{2}$ of two graphs $G_{1}$ and $G_{2}$ is defined to be the graph obtained by taking one copy of $G_{1}$ of order $p_{1}$ and $p_{1}$ copies of $G_{2}$ and joining $i^{\text {th }}$ vertex of $G_{1}$ with an edge to every vertex in the $i^{\text {th }}$ copy of $G_{2}$.

Definition 2.2: The graph $P_{n} \odot K_{1}$ is known as comb.

Theorem 2.3 $i^{e}\left(P_{n} \odot K_{1}\right)=\gamma^{e}\left(P_{n} \odot K_{1}\right)= \begin{cases}2 & \text { for } n=2 \\ 3 & \text { for } n=3 \\ 4 & \text { for } n=4 \\ i^{e}\left(P_{n-4}\right)+n & \text { for } n \geq 5\end{cases}$ 
Proof: Let $v_{1}, v_{2}, \ldots, v_{n}$ be the vertices of path $P_{n}$ and $u_{1}, u_{2}, \ldots, u_{n}$ are pendant vertices of comb $P_{n} \odot K_{1}$.

Case 1: $n=2$

In this case $N^{e}\left(u_{1}\right)=\left\{v_{1}\right\}, N^{e}\left(u_{2}\right)=\left\{v_{2}\right\}, N\left(v_{1}\right)=\left\{u_{1}, v_{2}\right\}, N^{e}\left(v_{2}\right)=\left\{v_{1}, u_{2}\right\}$. Therefore $D=\left\{u_{1}, u_{2}\right\}$ is an equi independent equitable dominating set with minimum cardinality. Hence, $i^{e}\left(P_{2} \odot K_{1}\right)=\gamma^{e}\left(P_{2} \odot K_{1}\right)=2$.

Case 2: $n=3$

In $P_{3} \odot K_{1}$, vertex $u_{2}$ is an equitable isolates and vertices $u_{1}, u_{3}$ are pendant vertices. Then $D=\left\{u_{2}, v_{1}, v_{3}\right\}$ is an equi independent equitable dominating set of $P_{3} \odot K_{1}$ with minimum cardinality. Hence, $i^{e}\left(P_{3} \odot K_{1}\right)=\gamma^{e}\left(P_{3} \odot K_{1}\right)=3$.

Case 3: $n=4$

In $P_{4} \odot K_{1}$, vertices $u_{2}, u_{3}$ are equitable isolates and $u_{1}, u_{4}$ are pendant vertices. Then $D=\left\{v_{1}, u_{2}, u_{3}, v_{4}\right\}$ is an equi independent equitable dominating set of $P_{4} \odot K_{1}$ with minimum cardinality. Hence, $i^{e}\left(P_{n} \odot K_{1}\right)=\gamma^{e}\left(P_{4} \odot K_{1}\right)=4$

Case 4: $n \geq 5$

In this case $u_{2}, u_{3}, \ldots, u_{n-1}$ are equitable isolates of $P_{n} \odot K_{1}$. Therefore the set $\left\{u_{2}, u_{3}, \ldots, u_{n-1}\right\}$ must be a subset of every equitable dominating set. While vertex $u_{1}$ is equitably adjacent to only $v_{1}$ and vertex $u_{n}$ is equitably adjacent to only $v_{n}$. Therefore one of the pair from $\left\{v_{1}, v_{n}\right\}$ or $\left\{u_{1}, u_{n}\right\}$ must belongs to every equitable dominating set which implies that $i^{e}\left(P_{n} \odot K_{1}\right) \geq i^{e}\left(P_{n-4}\right)+n$

Now depending upon the number of vertices of path $P_{n}$, consider the following subsets.

For $n \equiv 0(\bmod 3), D=\left\{v_{1} v_{n} v_{3 i+1}, u_{2}, u_{3}, \ldots, u_{n-1}\right\}$ where $0 \leq i \leq \frac{n}{3}-2$,

for, $n \equiv 1(\bmod 3) D=\left\{v_{1} v, v_{3 i+1}, u_{2}, u_{3}, \ldots, u_{n-1}\right\}$ where $0 \leq i \leq\left\lfloor\frac{n}{3}\right\rfloor-2$,

for, $n \equiv 2(\bmod 3), D=\left\{v_{1} v v_{3 i+1}, v_{n-2}, u_{2}, u_{3}, \ldots, u_{n-1}\right\}$ where $i=0,1, \ldots$ such that $3 i+1<n-1$.

In each of the above case $|D|=n+i^{e}\left(P_{n-4}\right)$. Observe that $N^{e}\left(v_{1}\right)=\left\{u_{1}, v_{2}\right\}, N^{e}\left(v_{n}\right)=\left\{u_{n}, v_{n-1}\right\}, \quad N^{e}\left(v_{3 i+1}\right)=\left\{v_{3 i}, v_{3 i+2}\right\}$ and $N^{e}\left(v_{n-2}\right)=\left\{v_{n-3}, v_{n-1}\right\}$. Therefore $N^{e}[D]=V\left(P_{n} \odot K_{l}\right)$ and $D$ is an equitable dominating set of $P_{n} \odot K_{1}$. Also $D$ is an equitable independent set as pendant vertices $u_{2}, u_{3}, \ldots, u_{n-1}$ are not equitable adjacent to any other vertex of $D$ and remaining vertices form $\gamma-$ set of $P_{n}$. Therefore $D$ is an equi independent equitable dominating set of $P_{n} \odot K_{1}$ with $|D|=n+i^{e}\left(P_{n-4}\right)$. Hence, $i^{e}\left(P_{n} \odot K_{1}\right)$ $=n+i^{e}\left(P_{n-4}\right)$.

Definition 2.4: The crown $C_{n} \odot K_{1}$ is obtained by joining pendant edge to each vertex of cycle $C_{n}$. 
Theorem 2.5: $\left.i^{e}\left(C_{n} \odot K_{1}\right)=\gamma^{e}\left(C_{n} \odot K_{1}\right)=\gamma^{e}\left(C_{n}\right)+n\right)$.

Proof: Let $v_{1}, v_{2}, \ldots, v_{n}$ be the vertices of degree 3 and $u_{1}, u_{2}, \ldots, u_{n}$ vertices of degree 1 of crown $C_{n} \odot K_{1}$. Observe that vertices $u_{1}, u_{2}, \ldots, u_{n}$ are equitable isolates of $C_{n} \odot K_{1}$. This implies that they must belong to every equitable dominating set which implies that $\left.i^{e}\left(C_{n} \odot K_{1}\right) \geq \gamma^{e}\left(C_{n} \odot K_{1}\right) \geq \gamma^{e}\left(C_{n}\right)+n\right)$.

Let $S$ be the $\gamma^{e}$ - set of $C_{n}$ and $D=\left\{u_{1}, u_{2}, \ldots, u_{n}\right\} \bigcup S$ with $|D|=\gamma^{e}\left(C_{n}\right)+n$. We claim that $D$ is an equi independent equitable dominating set of $C_{n} \odot K_{1}$. Observe that $D$ is an equitable dominating set of $C_{n} \odot K_{1}$ with minimum cardinality as all the equitable isolates belongs to $D$ and remaining all the vertices are dominated by set $S$. Also $D$ is an equitable independent set as all $u_{1}, u_{2}, \ldots, u_{n}$ are equitable isolates and vertices of set $S$ are not equitably adjacent to any other vertex of set $D$. Hence, $\left.i^{e}\left(C_{n} \odot K_{1}\right)=\gamma^{e}\left(C_{n} \odot K_{1}\right)=\gamma^{e}\left(C_{n}\right)+n\right)$.

Definition 2.6: The armed crown $A C r_{n}$ is a graph in which path $P_{2}$ is attached at each vertex of cycle $C_{n}$ by an edge.

Theorem 2.7: $i^{e}\left(A C r_{n}\right)=\gamma^{e}\left(A C r_{n}\right)=n$.

Proof: Let $w_{1}, w_{2}, \ldots, w_{n}$ be the pendant vertices, $u_{1}, u_{2}, \ldots, u_{n}$ be the vertices of degree 2 and $v_{1}, v_{2}, \ldots, v_{n}$ be the vertices of degree 3 of $A C r_{n}$. To dominate pendant vertices $w_{1}, w_{2}, \ldots, w_{n}$ equitably at least one of the $u_{i}$ or $w_{i}$ must belong to any equitable dominating set which implies that, $i^{e}\left(A C r_{n}\right) \geq n$

Let $D=\left\{u_{1}, u_{2}, \ldots, u_{n}\right\}$ with $|D|=n$. Note that $N^{e}[D]=V\left(A C r_{n}\right)$ and $\left|d\left(u_{i}\right)-d\left(v_{i}\right)\right|=$ $\left|d\left(u_{i}\right)-d\left(w_{i}\right)\right|=1$. Therefore $D$ is an equitable dominating set with minimum cardinality. Also $D$ is an equitable independent set as vertices of $D$ are non adjacent to each other. Therefore $D$ is an equi independent equitable dominating set with $|D|=n$. Hence, $i^{e}\left(A C r_{n}\right)=\gamma^{e}\left(A C r_{n}\right)=n$.

Definition 2.8: The gear graph $G_{n}$ is obtained from the wheel $W_{n}$ by subdividing each of its rim edge.

Theorem 2.9: $i^{e}\left(G_{n}\right)=\gamma^{e}\left(G_{n}\right)= \begin{cases}2 & \text { for } n=3 \\ 3 & \text { for } n=4 \\ i^{e}\left(C_{2 n}\right)+1 & \text { for } n \geq 5\end{cases}$

Proof: Let $v$ be the apex vertex, $v_{1}, v_{2}, \ldots, v_{n}$ be rim vertices, each vertex $u_{i}$ that subdividing an edge $v_{i} v_{i+1}$ for $1 \leq i \leq n-1$ and vertex $u_{n}$ subdividing an edge $v_{n} v_{1}$ of gear graph $G_{n}$. 
Case 1: $n=3$

Gear graph $G_{3}$ is not complete graph which implies that $i^{e}\left(G_{3}\right)>1$. Consider $D=\left\{v_{1}, u_{2}\right\}$ with $|D|=2$. Note that $D$ is an equi independent equitable dominating set. Hence, $i^{e}\left(G_{3}\right)=2$.

Case 2: $n=4$

Observe that $\left|d(v)-d\left(v_{i}\right)\right|=1=\left|d\left(v_{i}\right)-d\left(u_{j}\right)\right|$ where $v_{i} u_{j} \in E\left(G_{4}\right)$. Consider $D=\left\{v_{1}, u_{2}\right.$, $\left.u_{3}\right\}$ with $|D|=3$. Note that $N^{e}\left[v_{1}\right] \cup N^{e}\left[u_{2}\right] \cup N^{e}\left[u_{3}\right]=V\left(G_{4}\right)$ and vertices $v_{1}, u_{2}, u_{3}$ are non adjacent vertices. Therefore $D$ is an equi independent equitable dominating set of $G_{4}$. Hence, $i^{e}\left(G_{4}\right)=3$.

Case 3: $n \geq 5$

In this case apex vertex $v$ is an equitable isolates and set $V\left(G_{n}\right)-\{v\}$ form $C_{2 n}$. Let $S$ be the $i^{e}-$ set of $C_{2 n}$. Therefore $D=\{v\} \bigcup S$ is an equi independent equitable dominating set with minimum cardinality. Hence, $i^{e}\left(G_{n}\right)=i^{e}\left(C_{2 n}\right)+1$.

Definition 2.10: The fan $f_{n}$ is graph on $n+1$ vertices obtained by joining all the vertices of $P_{n}$ to a new vertex called apex vertex.

Theorem 2.11: $i^{e}\left(f_{n}\right)=\gamma^{e}\left(f_{n}\right)= \begin{cases}1 & \text { for } n=3 \\ 2 & \text { for } n=4 \\ i^{e}\left(P_{n}\right)+1 & \text { for } n \geq 5\end{cases}$

Proof: Let $v$ be a apex vertex and $v_{1}, v_{2}, \ldots, v_{n}$ be the rim vertices of fan $f_{n}$.

Case 1: $n=3$

In $f_{3}, D=\left\{v_{2}\right\}$ equitably dominate all the vertices and $D$ is an equitable independent set. Hence, $i^{e}\left(f_{3}\right)=1$.

Case 2: $n=4$

Observe that vertices $v_{2}$ and $v_{4}$ are non adjacent to each other. While $N^{e}\left[v_{2}\right] \cup N^{e}\left[v_{4}\right]=V\left(f_{4}\right)$. Hence, $D=\left\{v_{2}, v_{4}\right\}$ is an equi independent equitable dominating set of $f_{n}$ and $i^{e}\left(f_{3}\right)=2$.

Case 3: $n \geq 5$

In this case, apex vertex $v$ is equitable isolates and $V\left(f_{n}\right)-\{v\}$ form path $P_{n}$. Let $S$ be the $i^{e}$ - set of $P_{n}$. Therefore $D=\{v\} \bigcup S$ is an equi independent equitable dominating set with minimum cardinality. Hence, $i^{e}\left(f_{n}\right)=i^{e}\left(P_{n}\right)+1$.

Definition 2.12: The double fan $D f_{n}$ is given by $P_{n}+2 K_{1}$. 
Theorem 2.13: $i^{e}\left(D f_{n}\right)=\gamma^{e}\left(D f_{n}\right)= \begin{cases}1 & \text { for } n=2 \\ 1 & \text { for } n=3 \\ 2 & \text { for } n=4 \\ 2 & \text { for } n=5 \\ i^{e}\left(P_{n}\right)+2 & \text { for } n \geq 6\end{cases}$

Proof: Let $v_{1}, v_{n}$ be the vertices of degree 3, $u, v$ be the vertices of degree $n$ and the vertices $v_{2}, v_{3}, \ldots, v_{n-1}$ are of degree 4 of double fan $D f_{n}$.

Case 1: $n=2$

In $D f_{2}, D=\left\{v_{1}\right\}$ is an equi independent equitable dominating set of $D f_{2}$. Hence, $i^{e}\left(D f_{2}\right)=\gamma^{e}\left(D f_{2}\right)=1$.

Case 2: $n=3$

Observe that $D f_{3}$ is wheel $W_{4}$. Hence, $i^{e}\left(D f_{3}\right)=i^{e}\left(W_{3}\right)=1$.

Case 3: $n=4$

Observe that $N^{e}\left(v_{1}\right) \cup N^{e}\left(v_{4}\right)=V\left(D f_{4}\right)$ and $v_{1}, v_{4}$ are not adjacent vertices. Also none of the vertex is equitably adjacent to every vertex of $D f_{4}$. Therefore $D=\left\{v_{1}, v_{4}\right\}$ is an equi independent equitable dominating set of $D f_{4}$. Hence, $i^{e}\left(D f_{4}\right)=\gamma^{e}\left(D f_{4}\right)=2$.

Case 4: $n=5$

In $D f_{5}$, no vertex is adjacent to all the vertices of $D f_{5}$. Consider $D=\left\{v_{2}, v_{4}\right\}$. Observer that $D$ is an equi independent equitable dominating set and $D$ is an independent set of $D f_{5}$ with $|D|=2 . i^{e}\left(D f_{5}\right)=\gamma^{e}\left(D f_{5}\right)=2$.

Case 5: $n \geq 6$

In this case $u$ and $v$ are equitable isolates and $V\left(D f_{n}\right)-\{u, v\}$ form path $P_{n}$. Let $S$ be the $i^{e}-$ set of $P_{n}$. Therefore $D=S \bigcup\{u, v\}$ is an equi independent equitable dominating set of $D f_{n}$ with minimum cardinality. Hence, $i^{e}\left(D f_{n}\right)=i^{e}\left(P_{n}\right)+2$.

Definition 2.14: The flower $F l_{n}$ is the graph obtained from a helm $H_{n}$ by joining each pendant vertex to the apex of the helm.

Theorem 2.15: $i^{e}\left(F l_{n}\right)=\gamma^{e}\left(C_{n}\right)+n+1$.

Proof: Let $v$ be the apex vertex $, u_{1}, u_{2}, \ldots, u_{n}$ be the vertices of degree 2 and $v_{1}, v_{2}, \ldots, v_{n}$ be the vertices of degree 4 of $F l_{n}$. Observe that vertices $v, u_{1}, u_{2}, \ldots, u_{n}$ are equitable isolate of $F l_{n}$. Therefore they must belong to every equitable dominating set of $F_{n}$ which implies that $i^{e}\left(F l_{n}\right) \geq \gamma^{e}\left(C_{n}\right)+n+1$. 
Let $S$ be the $i^{e}-$ set of $C_{n}$ and $D=\left\{v, u_{1}, u_{2}, \ldots, u_{n}\right\} \bigcup S$ with $|D|=i^{e}\left(C_{n}\right)+n+1=\gamma^{e}\left(C_{n}\right)+$ $n+1$. Observe that $N^{e}[D]=V\left(F l_{n}\right)$. Therefore $D$ is an equitable dominating set of $F l_{n}$. Also $D$ is an equitable independent set of $F l_{n}$ as $v, u_{1}, u_{2}, \ldots, u_{n}$ are equitable isolates and vertices of $S$ are non adjacent to each other. Hence, $D$ is an equi independent equitable dominating set of $F l_{n}$ and $i^{e}\left(F l_{n}\right)=\gamma^{e}\left(C_{n}\right)+n+1$.

Definition 2.16: A one point union $C_{n}^{(k)}$ of $k$ copies of cycle $C_{n}$ is the graph obtained by taking $v$ as a common vertex such that any two cycles $C_{n}^{(i)}$ and $C_{n}^{(i)}(i \neq j)$ are edge disjoint and do not have any vertex in common except $v$.

Definition 2.17: The friendship graph $F_{n}$ is a one-point union of $n$ copies of cycles $C_{3}$.

Theorem 2.18: $i^{e}\left(F_{n}\right)=\gamma^{e}\left(F_{n}\right)=n+1$

Proof: Let $v$ be the apex vertex and $v_{1}, v_{2}, \ldots, v_{2 n}$ be other vertices of $F_{n}$. Here $v$ is an equitable isolates of $F_{n}$. Therefore $v$ must belong to every equitable dominating set of $F_{n}$. Also vertex $v_{i}$ is equitably adjacent to only $v_{i+1}$ and vice versa, where $i=1,3, \ldots, 2 n-1$ which implies that at least one vertex from each pair $\left\{v_{i}, v_{i+1}\right\}$ must belong to every equitable dominating set of $F_{n}$. Therefore $D=\left\{v, v_{1}, v_{3}, \ldots, v_{2 n-1}\right\}$ is an equitable dominating set as well as it is an independent set of $F_{n}$. Hence, $D$ is an equi independent equitable dominating set of $F_{n}$ and $i^{e}\left(F_{n}\right)=\gamma^{e}\left(F_{n}\right)=n+1$.

\section{Concluding Remarks}

Some fundamental results on the concept of equi independent equitable domination number are established by the authors but for the sake of brevity they are not reported here. Here we investigated equi independent equitable domination number for some special graphs. To establish the bounds in terms of various graph theoretic parameters in the context of equi independent equitable domination number is an open area of research.

\section{Acknowledgement}

The authors are highly thankful to the anonymous referees for careful reading of first two drafts of this paper.

\section{References}

1. B. Basavanagoud and S. M. Hosamani, J. Sci. Res. 3(3), 547 (2011). http://dx.doi.org/10.3329/jsr.v3i3.7744

2. C. Berge, Theory of Graphs and its Applications (Methuen, London, 1962).

3. E. J. Cockayne and S. T. Hedetniemi, Networks, 7, 247 (1977). http://dx.doi.org/10.1002/net.3230070305 
4. W. Goddard and M. A. Henning, Discrete Math. 313, 839 (2013).

http://dx.doi.org/10.1016/j.disc.2012.11.031

5. T. W. Haynes, S. T. Hedetniemi, and P. J. Slater, Fundamentals of Domination in Graphs, (Marcel Dekker, New York, 1998).

6. O. Ore, Amer. Math. Soc. Transl. 38, 206212, (1962).

7. V.Swaminathan and K. Dharmalingam, Kragujevac J. Math. 35(1), 191 (2011).

8. S. K. Vaidya and N. J. Kothari, Equi Independent Equitable Dominating Sets in Graphs, communicated for publication.

9. D. B. West, Introduction to Graph Theory, $2^{\text {nd }}$ Edition (Prentice-Hall, New Delhi, India, 2003). 